\title{
Deformation of P Cygni line profiles by gravitational microlensing effects
}

\author{
D. Hutsemékers
}

2004

\begin{abstract}
This short report provides details on the computation of deformations of P Cygni line profiles induced by gravitational microlensing effects, as well as a series of examples. This report supplements the results discussed in Hutsemékers et al. (1994).
\end{abstract}

\section{The formalism}

We consider a spherically symmetric atmosphere expanding around a central core, the source of the continuum. The flow is accelerated outwards. In the framework of the Sobolev approximation, the source function as well as the total optical depth may be evaluated locally in each point of the envelope. Measured by the observer, the normalized line profile at a frequency $\nu$ is simply expressed by (cf. Hutsemékers 1993):

$$
F_{\nu}=\int_{\Sigma_{\nu}} \frac{S_{\nu}}{I_{\nu}^{c}}\left(1-e^{-\tau_{\nu}}\right) 2 p d p+\int_{\Sigma_{\nu}} e^{-\tau_{\nu}} 2 p d p
$$

where $S_{\nu}$ is the Sobolev source function, $\tau_{\nu}$ the Sobolev optical depth, $I_{\nu}^{c}$ the intensity of the continuum emitted by the core, and $p$ the impact parameter expressed in units of the core radius (see Figure 1). The integration is performed over the so-called surfaces of equal frequency $\nu$, denoted $\Sigma_{\nu}$.

The magnification of the source by a microlens located along the line of sight can be accounted for by simply multiplying each elementary emitting surface in the source plane by an amplification factor $A$ which depends on the adopted microlens model and the projected distance between the lens and the core. Since the amplification is not symmetric with respect to the quasar center, the line profile function then reads

$$
F_{\nu}=\frac{\iint S_{\nu}\left(1-e^{-\tau_{\nu}}\right) A(p, \theta) p d p d \theta+\iint I_{\nu}^{c} e^{-\tau_{\nu}} A(p, \theta) p d p d \theta}{\iint I_{\nu}^{c} A(p, \theta) p d p d \theta}
$$

which simplifies to

$$
F_{\nu}=\int_{0}^{p_{\max }} \frac{S_{\nu}}{I_{\nu}^{c}}\left(1-e^{-\tau_{\nu}}\right) \frac{A^{\star}(p)}{A_{c}^{\star}} 2 p d p+\int_{0}^{1} e^{-\tau_{\nu}} \frac{A^{\star}(p)}{A_{c}^{\star}} 2 p d p
$$

if we define

$$
\begin{aligned}
A^{\star}(p) & =\frac{1}{2 \pi} \int_{0}^{2 \pi} A(p, \theta) d \theta \\
A_{c}^{\star} & =\int_{0}^{1} A^{\star}(p) 2 p d p .
\end{aligned}
$$




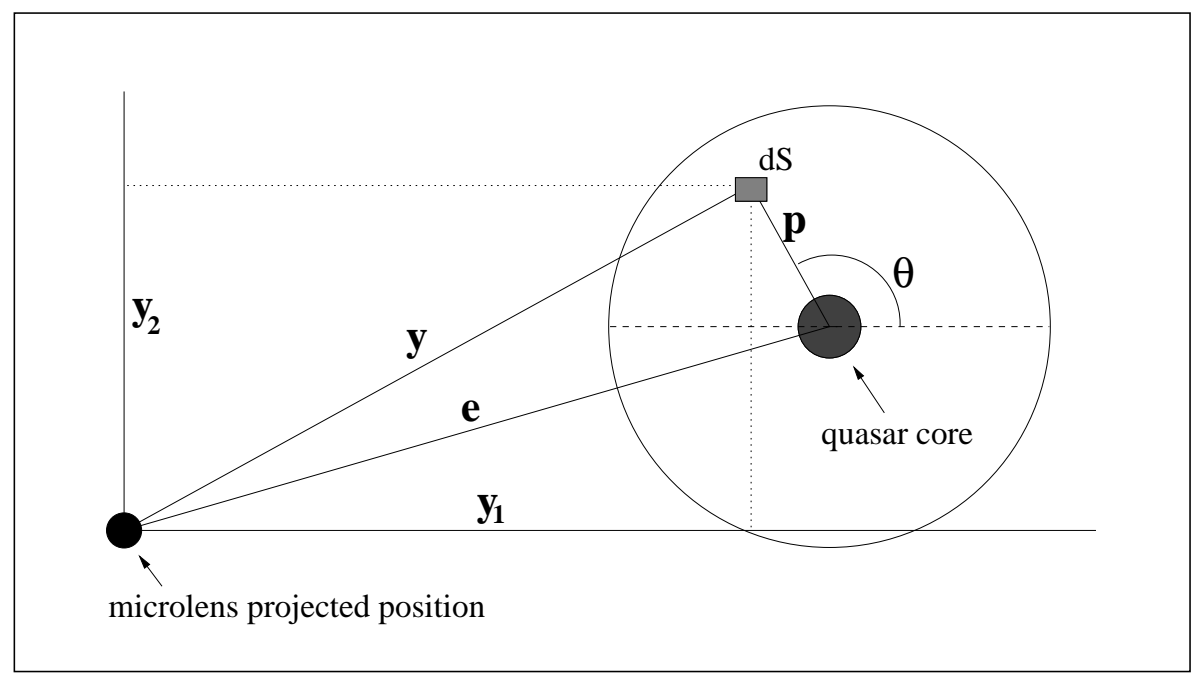

Figure 1: The geometry in the source plane.

The amplification factor $A(p, \theta)$ depends on the adopted microlens model. A generic model is the so-called Chang-Refsdal microlens which is basically a point-mass lens perturbed by the gravitational field of the lens galaxy. The amplification factor may be computed ${ }^{1}$ in each point of the source plane as a function of several parameters. It shows typical caustic patterns like those illustrated in the series of figures below. The amplification factor also depends on the size of the quasar core relative to the microlens effective radius in the source plane (the so-called Einstein radius, projected onto the source plane), and on their relative distance. By moving the quasar within such a caustic pattern, different amplification factors apply to different sub-regions of the wind, inducing deformations of the P Cygni line profiles.

\section{Numerical calculations}

A series of numerical calculations are given in the following figures. Each figure shows the track in the caustic pattern, the photometric light curve, the equivalent width curve, and 3 examples of P Cygni line profiles taken at different epochs along the light curve (cf. the tick marks between the flux and equivalent width diagrams).

The first two pages of figures illustrate the effect of different tracks and/or caustic patterns. For each case, two different values of the core radius (expressed in units of the Einstein radius) are considered. The same outflow model is used (i.e. a typical P Cygni type model with pure resonance scattering and a smooth opacity law).

The last page illustrates the effect of microlensing on different outflow models, i.e. a model with significant collisional excitation, a model with an opacity shell inside the flow, and a model with both the shell and the collisional excitation.

\section{Results}

It is clear from the illustrated profiles that microlensing can induce significant deformations of P Cygni line profiles. Both emission and absorption may be enhanced.

\footnotetext{
${ }^{1}$ See Schneider et al. (1992) for a complete derivation of the amplification factor in the case of a ChangRefsdal microlens
} 
In some cases, the modified profiles can even mimic pure emission or pure absorption. It is important to notice that when deformations are significant, all velocities are affected such that only global variations are seen (as opposed to the possible appearance of narrow peaks in the profiles for example).

The enhancement of the emission part of the profile is rather unexpected since emission is usually thought to come from regions much larger than the continuum source (and consequently much larger than the Einstein radius). This enhancement may be explained by the fact that the source function is large close to the core, rapidly decreasing outwards; the amplification of the emission then occurs just before or after the magnification of the continuum source.

Whatever the caustic pattern or the outflow model, the profile deformations and the equivalent width curves show a characteristic behavior. Also, the first order moment $W_{1}$ of the line profiles (e.g. Hutsemékers \& Surdej, 1990) is found to remain constant whatever the deformations induced by the microlensing effect. This important property may be easily understood: along a given line of sight, microlensing amplification similarly affects positive and negative velocities in the profile, producing symmetric variations which cancel each other when computing $W_{1}$.

\section{References}

- Hutsemékers, D., 1993, ApJ 417, 97

- Hutsemékers, D., Surdej, J., 1990, ApJ 361, 367

- Hutsemékers, D., Surdej, J., Van Drom E., 1994, Ap\&SS 216, 361

- Schneider, P., Ehlers, J., Falco, E., 1992, Gravitational lenses (Springer, Berlin). 

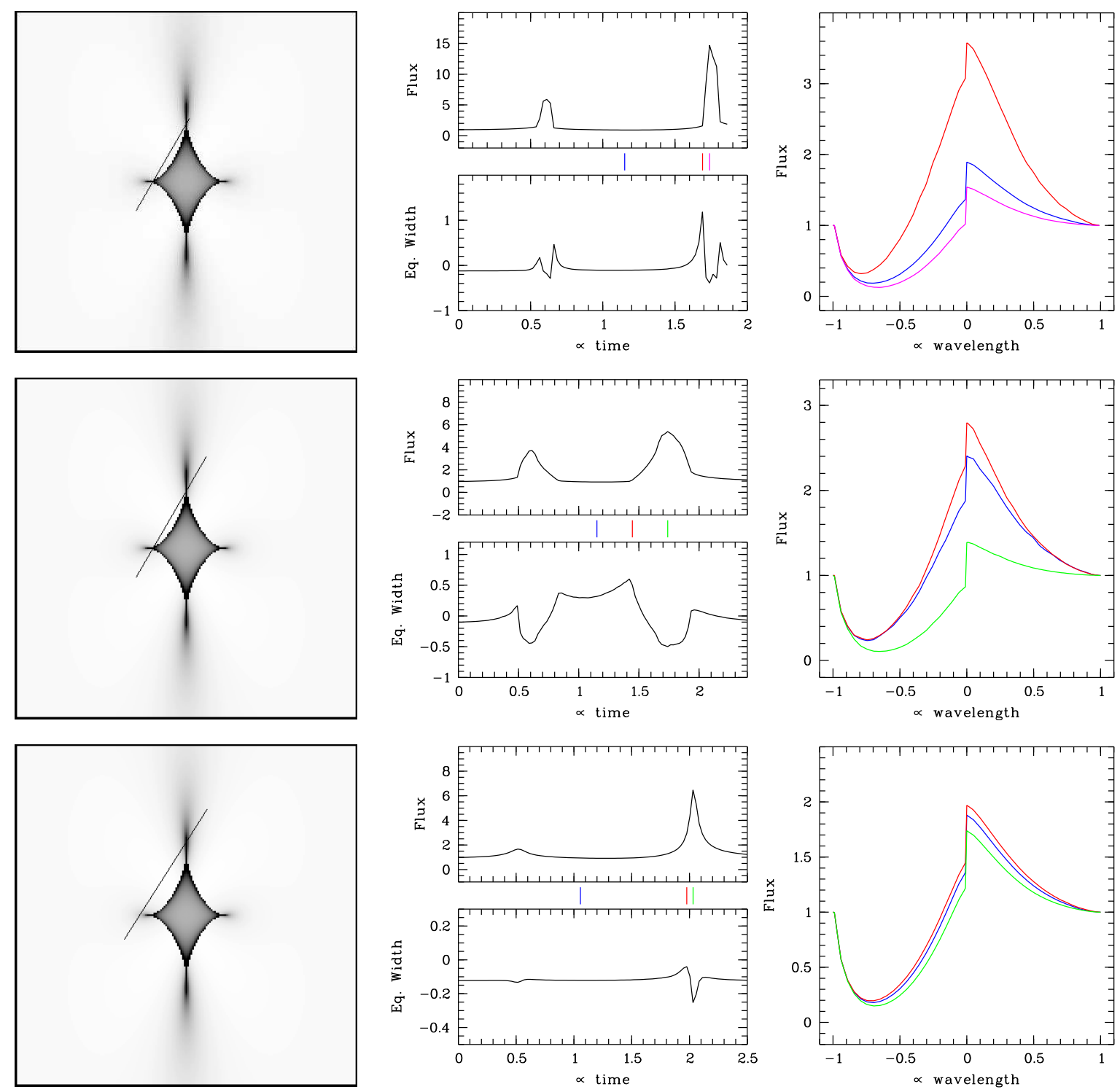

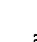
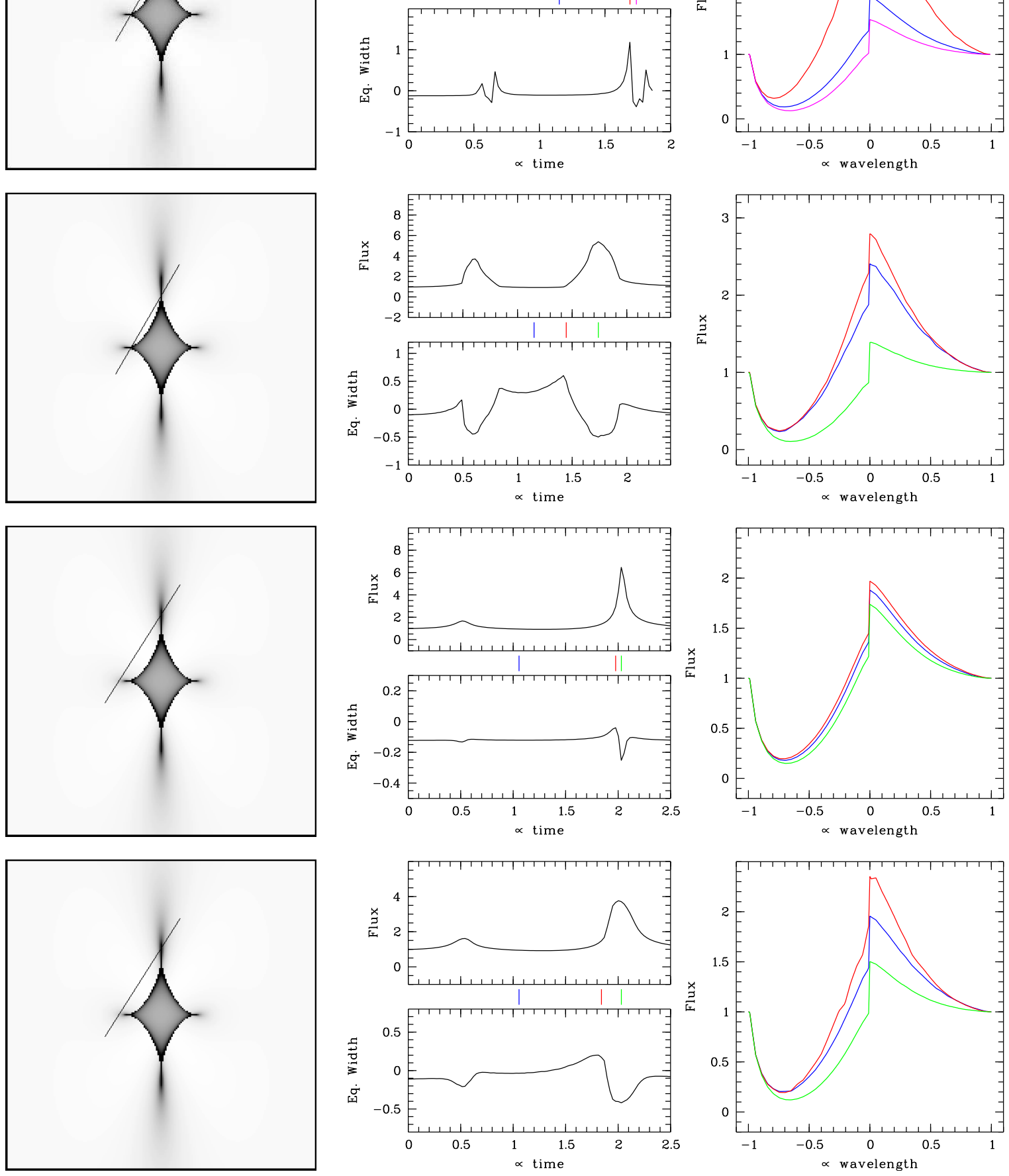

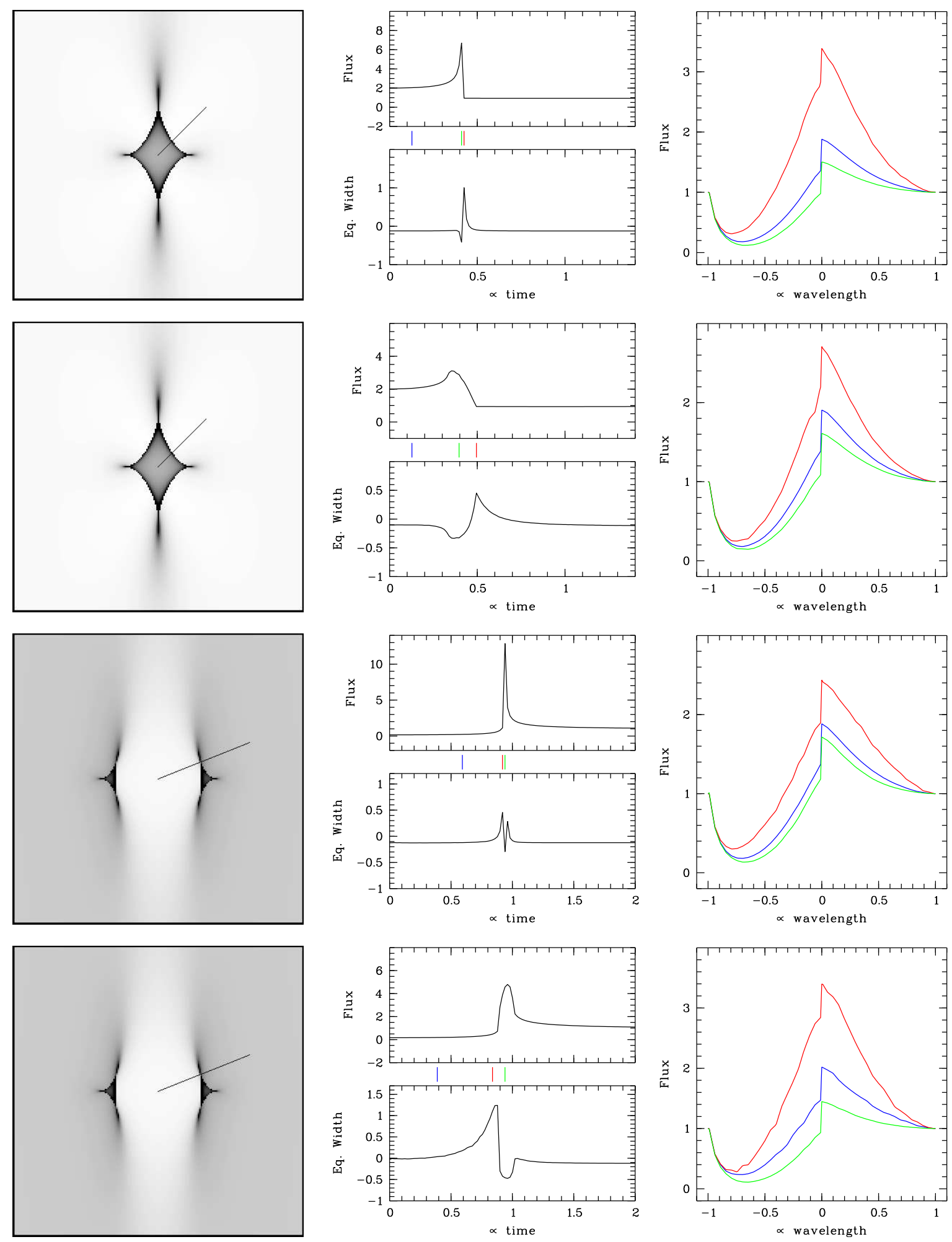

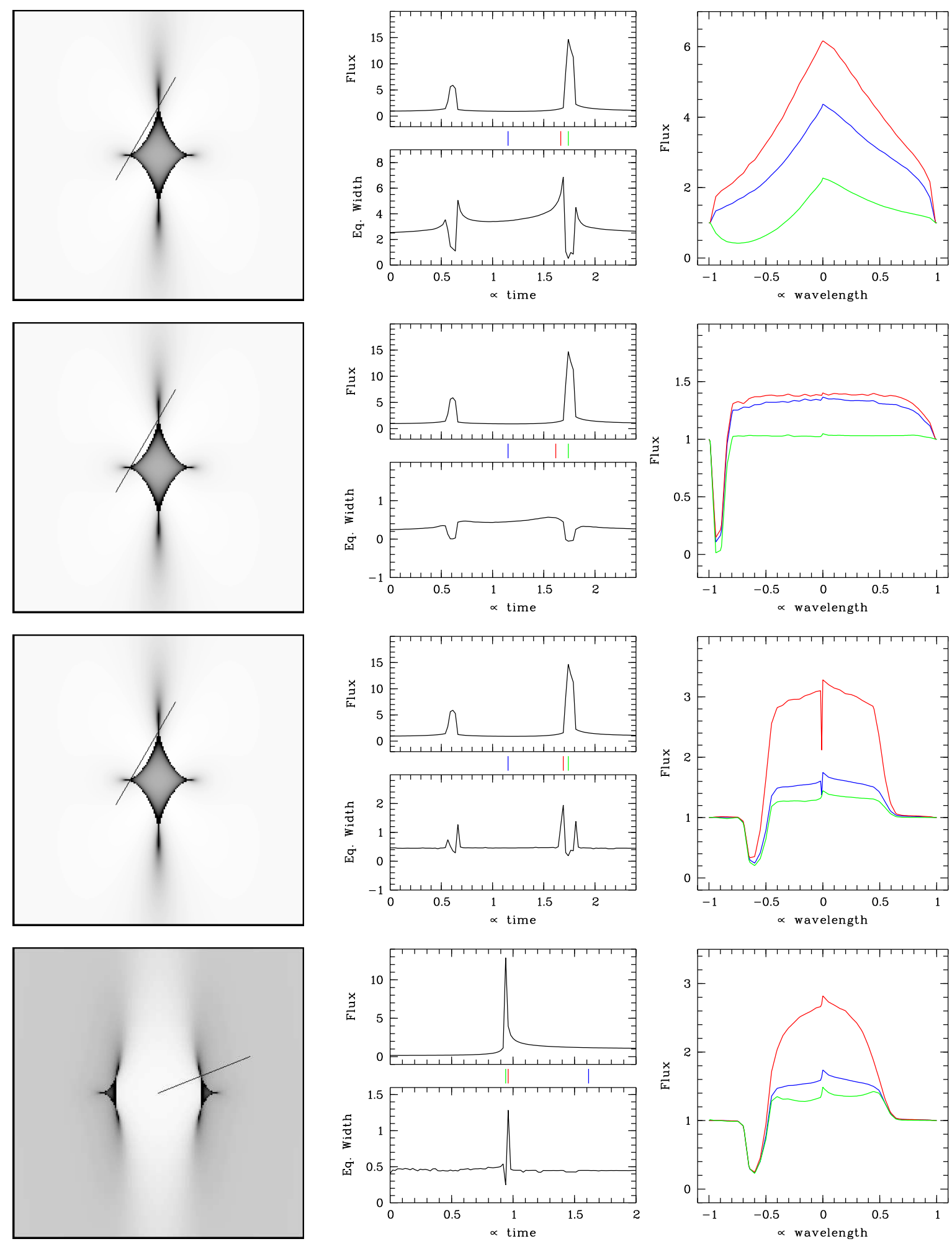